\title{
Peignes de fréquences auto-référencés pour la mesure de fréquences optiques
}

\author{
Yann LE COQ, Rodolphe LE TARGAT \\ LNE-SYRTE, CNRS/UPMC/Observatoire de Paris, 61 avenue de l'Observatoire, 75014 Paris \\ yann.lecog@obspm.fr
}

L'avènement des peignes de fréquences, au début des années 2000, a révolutionné la métrologie des fréquences optiques (i.e. quelques centaines de $\mathrm{THz}$ ), et a stimulé le développement d'une nouvelle génération d'horloges atomiques, référencées sur des transitions optiques. Ces peignes, basés sur des lasers à impulsions femtosecondes, sont aujourd'hui le moyen le plus efficace et fiable de réaliser des comparaisons de fréquences entre une horloge optique et des étalons primaires du domaine micro-onde, ainsi qu'entre deux horloges optiques fonctionnant à des fréquences différentes. Nous présentons ici les concepts principaux de ces nouveaux outils, dont les applications s'étendent aujourd'hui bien au-delà de la communauté métrologique pure et dure.

es horloges atomiques fonctionnant

- dans le domaine micro-onde (i.e. à une fréquence de quelques $\mathrm{GHz}$ ), sur lesquelles se basent les réalisations de l'unité SI de la seconde et le temps atomique international atteignent aujourd'hui leurs limites, et les systèmes fonctionnant dans le domaine optique sont, à terme, amenés à les remplacer. Ces derniers dispositifs fonctionnent en interrogeant une transition atomique hyper étroite à l'aide d'un laser de très haute stabilité et pureté spectrale. En asservissant le laser de sorte qu'il reste à résonance avec la transition atomique sondée, on réalise ainsi un étalon de fréquence dans le domaine optique (i.e. à une fréquence de quelques centaines de $\mathrm{THz}$ ). La stabilité en fréquence de ces étalons optiques (quelques 10-15 à 1 seconde actuellement) surpasse largement celle des étalons de fréquences micro-ondes (à base de cesium ou de rubidium), ce qui constitue une très forte motivation pour leur développement. Par ailleurs, une meilleure stabilité est également un moyen de caractériser les biais systématiques pouvant impacter un étalon de fréquence beaucoup plus rapidement et précisément. En termes d'exactitude, de nombreux effets sont insensibles à la fréquence de référence (effet Zeeman, effet de densité par exemple), et deviennent donc beaucoup plus petits en valeur relative, lorsqu'ils sont rapportés à une fréquence optique plutôt que micro-onde. Seuls les effets liés au mouvement (effet Doppler, effet de recul) dépendent de la fréquence, mais ce point particulier a été résolu pour les horloges optiques soit en confinant les particules dans un réseau optique (atomes neutres), soit dans un piège à ions (particules chargées).

L'avantage lié à l'utilisation des étalons de fréquences optiques est donc particulièrement clair. L'inconvénient réside dans le fait que le signal fourni par ce type d'horloge est un champ électromagnétique optique, dont la fréquence d'oscillation est de plusieurs centaines de $\mathrm{THz}$ : aucun système électronique n'est suffisamment rapide pour le compter ou le comparer à d'autres étalons de fréquences, qu'ils soient optiques (à d'autres longueurs d'ondes) ou micro-ondes.

Avant l'invention du peigne de fréquences, la seule solution pour effectuer des mesures de fréquences optiques passait par des chaînes de multiplication de fréquences successives extrêmement complexes, impliquant des dizaines de lasers, et très lourdes à mettre en œuvre, à tel point que seule une poignée de laboratoires au monde possédait une telle technologie. Les peignes de fréquences, générés par des lasers femtosecondes auto-référencés, permettent aujourd'hui de réaliser une telle mesure avec un dispositif simple, très compact (environ $1 \mathrm{~m}^{2}$ de table optique), fiable, et désormais disponible commercialement.

\section{Principe d'un peigne de fréquences optiques}

La lumière émise par un laser femtoseconde à mode bloqué se compose (comme celui représenté sur la figure 1), dans le domaine fréquentiel, d'une série de modes régulièrement espacés en fréquence et cohérents en phase les uns avec les autres. La fréquence de chaque mode suit ainsi la relation : $\mathrm{v}_{\mathrm{N}}=\mathrm{Nf}_{\text {rep }}+\mathrm{f}_{0^{\prime}}$ où $f_{\text {rep }}$ est la cadence de répétition (radio ou basse fréquence) des impulsions laser, $v_{N}$ la fréquence optique du mode considéré, $\mathrm{N}$ un grand nombre entier, typiquement $10^{6}$, et $\mathrm{f}_{0}$ est une fréquence de décalage globale appelée «fréquence de décalage entre enveloppe et porteuse » (" carrier-envelop offset frequency » en anglais). Ce dernier terme est lié à la différence entre vitesse de phase et vitesse de groupe au sein de l'oscillateur laser et correspond, dans le domaine temporel, au fait que le champ électrique entre deux impulsions successives qui sortent de la cavité laser n'est pas strictement identique. Il présente un glissement de phase, 
par rapport à l'enveloppe temporelle des impulsions (figure 2).

En mélangeant sur une lame partiellement réfléchissante les champs électriques issus d'un laser femtoseconde et d'un laser continu dont on veut connaître la fréquence optique absolue $v_{\mathrm{cw}^{\prime}}$ et en détectant le résultat avec une photodiode rapide (de bande passante $\mathrm{f}_{\text {BW }}$ ), on obtient un signal radiofréquence dont le spectre se compose d'une part d'harmoniques de la cadence de répétition $\mathrm{f}_{\text {rep }}$ et d'autre part des différents battements optiques de fréquences $\mathrm{f}_{\mathrm{b}, \mathrm{N}}=\left|\mathrm{v}_{\mathrm{cw}}-\mathrm{Nf}_{\text {rep }}-\mathrm{f}_{0}\right|$, avec des

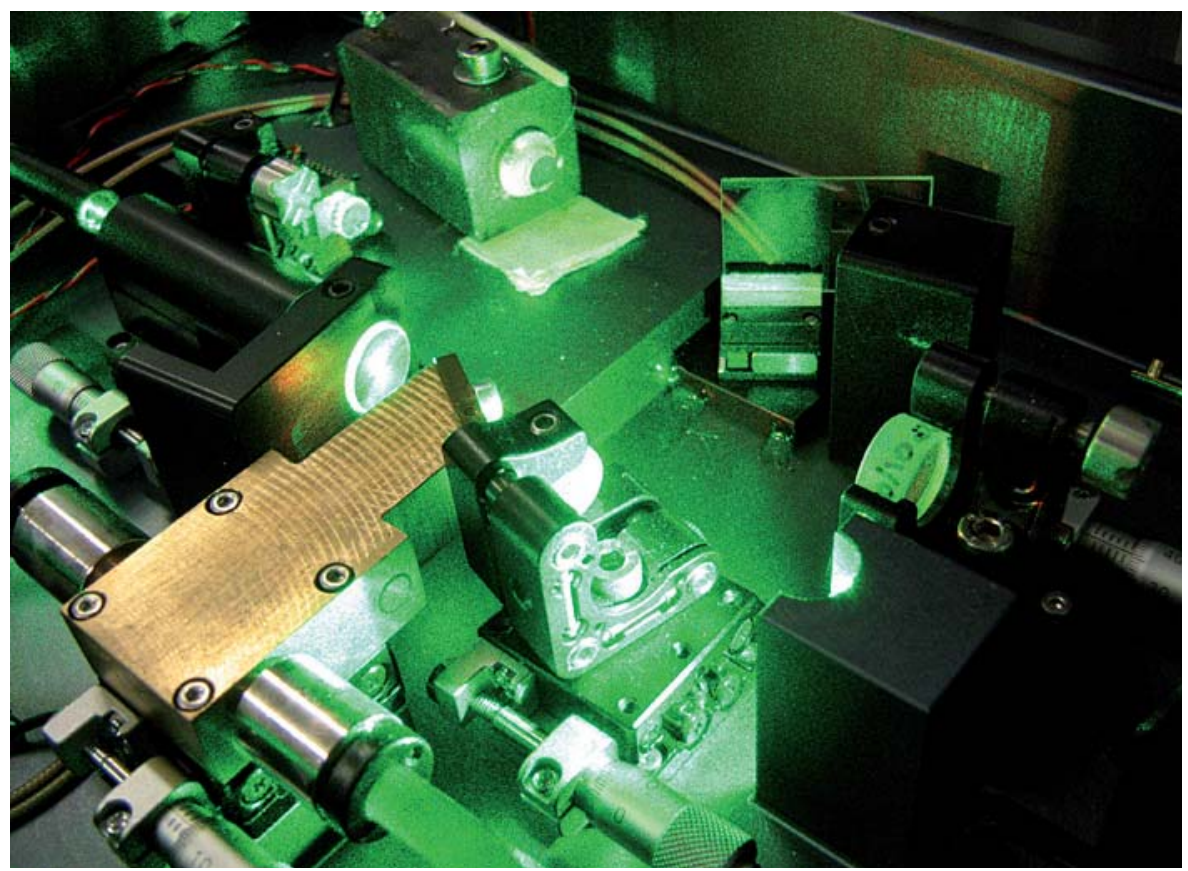

Figure 1. Photographie d'un laser femtoseconde (à base de titane-saphir) utilisé pour réaliser un peigne de fréquences optiques. Les applications de peigne de fréquences, préférant des dents du peigne bien espacées les unes des autres (donc plus faciles à identifier) et présentant une intensité par mode assez forte, privilégient des oscillateurs lasers femtosecondes à haute cadence de répétition (>100 MHz, $800 \mathrm{MHz}$ pour l'illustration).

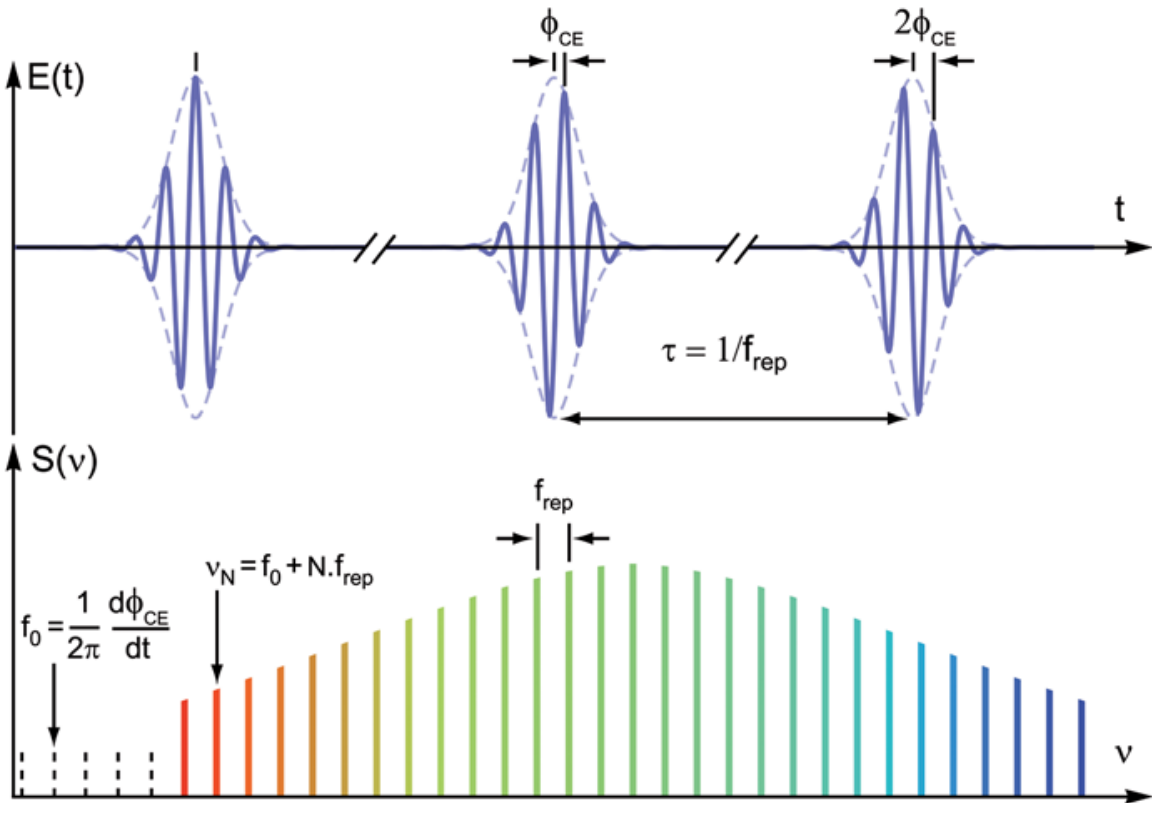

Figure 2. Correspondance entre le domaine temporel (impulsions successives) et fréquentiel (modes régulièrement espacés) pour le champ électrique issu d'un laser femtoseconde à modes bloqués. Le déphasage entre l'enveloppe et la porteuse suivant deux impulsions successives correspond, dans le domaine spectral, à un décalage global des dents d'une fréquence $f_{0}$.

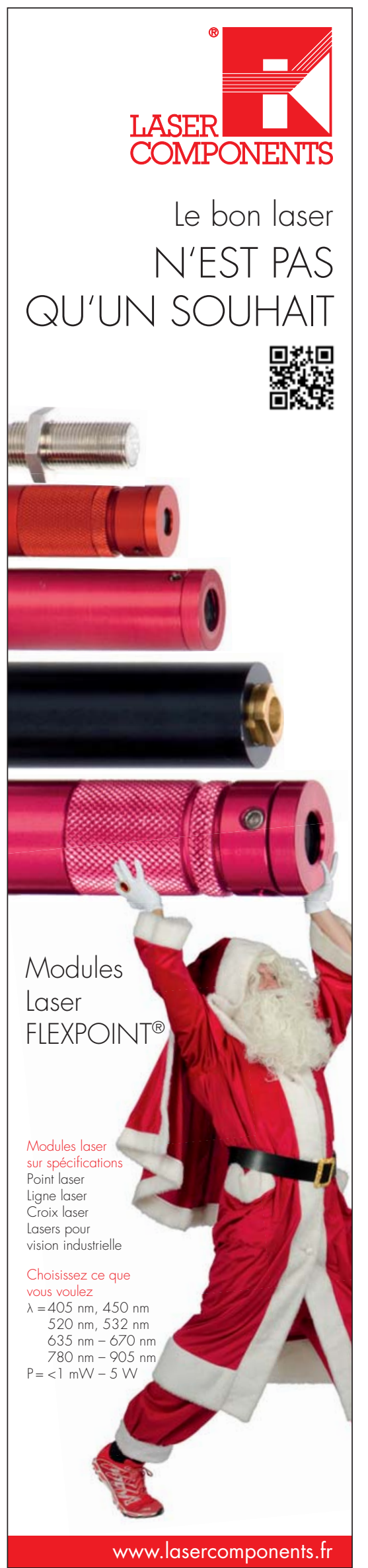

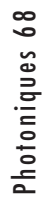


valeurs de l'entier $\mathrm{N}$ telles que $\mathrm{f}_{\mathrm{b}, \mathrm{N}}<\mathrm{f}_{\mathrm{BW}}$. On peut alors facilement isoler de ce signal complexe, à l'aide d'un filtre radiofréquence passe-bande suffisamment étroit, l'une de ces composantes $\mathrm{f}_{\mathrm{b}, \mathrm{Ncw}}$ qui fournit ainsi «l'écart en fréquence » du laser continu à mesurer avec l'un des modes du laser femtoseconde référencé par l'entier $\mathrm{N}_{\mathrm{cw}}$. Connaître de manière absolue la fréquence de ce mode particulier permet donc en principe de remonter à celle du laser à mesurer.

De la même façon, à l'aide d'un autre filtre passe-bande, on peut également isoler l'une des harmoniques de la cadence de répétition $\mathrm{f}_{\text {rep' }}$ ce qui donne accès à cette valeur. En pratique, il est cependant fréquent de séparer physiquement les fonctions de mesure de $f_{b, N c w}$ et $f_{\text {rep }}$ sur deux photodiodes différentes, afin d'optimiser les conditions de détection pour ces deux signaux séparément.

Si l'on suppose que l'entier $\mathrm{N}_{\mathrm{cw}}$ est connu, il reste uniquement la fréquence $f_{0}$ à déterminer pour contraindre l'ensemble du problème, et ainsi connaître absolument la fréquence optique $v_{c w}$ à mesurer.

\section{Auto-référencement}

Cette détermination de $\mathrm{f}_{0}$ se fait par la technique de l'auto-référencement (figure 3), qui présuppose que le laser femtoseconde ait un spectre couvrant au moins une octave (d'une fréquence optique fà sa fréquence double $2 f$ ). En pratique, très peu de lasers possèdent cette propriété (seuls les lasers titane-saphir à impulsions ultra-courtes de 3 à 5 femtosecondes environ présentent un spectre aussi étendu « naturellement »). Néanmoins, I'utilisation d'une fibre optique à haute non-linéarité permet, pour de nombreux systèmes, d'étendre le spectre jusqu'à la largeur nécessaire, tout en conservant les propriétés de cohérence nécessaires au fonctionnement du dispositif. Ces fibres optiques à haute non-linéarité sont ainsi conçues pour présenter une dispersion chromatique quasi nulle autour de la longueur d'onde centrale du laser femtoseconde, afin que les impulsions puissent s'y propager en restant très courtes et donc y conservent une forte intensité crête, gage de forts effets non-linéaires.

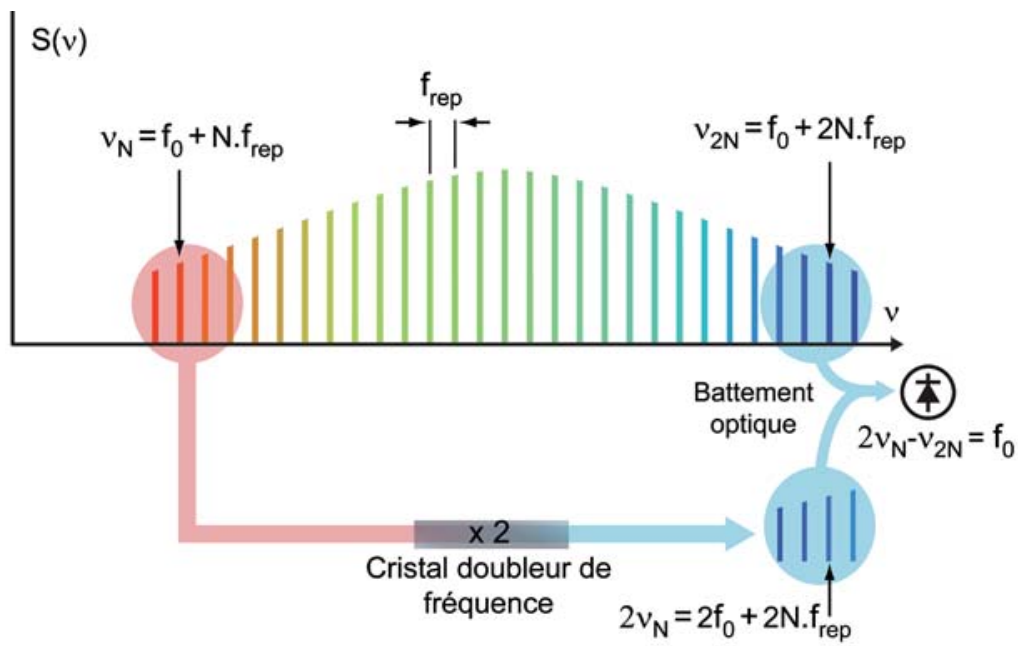

Figure 3. Schéma de principe de l'auto-référencement par la méthode f-2f. Le battement optique entre l'extrémité « rouge » du peigne de fréquences doublée et l'extrémité « bleve » fournit un signal présentant une composante à la fréquence $f_{0}$, qu'il est facile d'isoler à l'aide d'un filtre radiofréquence passe-bande étroit. On peut noter que plusieurs paires de modes doivent combiner leurs signaux de façon cohérente pour obtenir un signal à fréquence $f_{0}$ de grand rapport signal à bruit.

Par mélanges à quatre ondes (éventuellement dégénérés) successifs entre les différents modes composant le spectre du laser femtoseconde, la fibre élargit ainsi progressivement le spectre jusqu'à atteindre un rapport de fréquences optiques supérieur ou égal à 2 entre ses deux extrémités, nécessaire pour la méthode d'auto-référencement. Il apparaît ainsi dans le spectre une composante de fréquence optique $\mathrm{v}_{\mathrm{N}}=\mathrm{Nf}_{\text {rep }}+\mathrm{f}_{0}$ et une de fréquence $v_{2 \mathrm{~N}}=2 \mathrm{Nf}_{\text {rep }}+\mathrm{f}_{0}$ à l'autre bout $\mathrm{du}$ spectre. En doublant la fréquence de la composante $v_{N}$ à l'aide d'un cristal non-linéaire, et en produisant le signal de battement optique entre cette composante doublée, de fréquence $2 v_{N}$ et la composante de fréquence $v_{2 N}$ sur une photodiode rapide, on obtient ainsi un signal à la fréquence $\left|2 \mathrm{Nf}_{\text {rep }}+2 \mathrm{f}_{0}-2 \mathrm{Nf}_{\text {rep }}-\mathrm{f}_{0}\right|=\left|\mathrm{f}_{0}\right|$. Le doublement de fréquence agit sur plusieurs raies optiques $v_{N}$ simultanément, le signal obtenu présente donc également des harmoniques à des fréquences différentes de $\left|\mathrm{f}_{0}\right|$ qu'il est cependant très facile de supprimer à l'aide d'un filtre passebande radiofréquence suffisamment étroit. Précisons également que la participation d'un grand nombre de paires de modes de fréquences $2 v_{N}$ et $v_{2 N}$ au signal est en réalité tout à fait favorable puisque plusieurs paires verront leur battement optique s'additionner de façon cohérente afin de produire une composante de fréquence $\mathrm{f}_{0} \mathrm{~d}$ 'amplitude bien supérieure à ce que donnerait une unique paire de modes. La puissance optique par mode étant typiquement de l'ordre de quelques picowatts ou nanowatts, on voit bien là l'intérêt de ce processus de sommation cohérente. La sommation cohérente des signaux de fréquence $\mathrm{f}_{0}$ issus de paires de modes $\left(2 v_{N^{\prime}} v_{2 N}\right)$ ne peut néanmoins se produire que si la phase des signaux produits par chaque paire est identique, ce qui nécessite un ajustement fin de la dispersion globale des systèmes optiques réalisant cet interféromètre $f-2 f$ (longueur optique effective traversée par les composantes de fréquences $v_{2 N}$ et celle traversée par les composantes de fréquence $v_{N}$ avant, pendant et après doublage de fréquence). Cette condition d'accord de phase est en fait équivalente, dans le domaine temporel, à s'assurer que la partie de l'impulsion qui a été doublée en fréquence, et celle qui ne l'a pas été, arrivent simultanément sur la photodiode de détection du battement. Cette condition peut, par exemple, être simplement réalisée à l'aide d'une ligne à retard ajustable n'agissant que sur une partie du spectre optique (avant doublage de fréquence), ou bien par des éléments de dispersion fixe (lame de verre ou miroirs à dispersion négative) ad-hoc, ajoutés dans le trajet de propagation du faisceau. 


\section{Mesure de fréquence optique}

L'ensemble des signaux radiofréquences $f_{b, N c w^{\prime}} f_{r e p}$ et $f_{0}$ fournit donc l'intégralité des paramètres déterminant le système, et l'on a ainsi un dispositif permettant de comparer les fréquences optiques aux fréquences micro-ondes, plus traditionnelles en métrologie temps-fréquence. En outre, en disposant de plusieurs battements entre le peigne et différents lasers continus (à différentes longueurs d'ondes), $f_{b, N c w 1}, f_{b, N c w 2}, f_{b, N c w 3^{\prime}}$ etc. il est possible de comparer simultanément plusieurs fréquences optiques, sans nécessairement passer par l'intermédiaire des références micro-ondes.

Ces mesures présupposent néanmoins que les indices des modes $\mathrm{N}_{\mathrm{cw}(\mathrm{i})}$ soient connus. Le moyen le plus simple pour effectuer cette détermination est que la fréquence optique à mesurer soit préalablement connue (par exemple avec un lambda-mètre de très haute précision, ou bien s'il s'agit d'une transition atomique ou moléculaire très bien caractérisée dans la littérature) avec une exactitude supérieure à la cadence de répétition du peigne de fréquence $f_{\text {rep }}$. En effet, dans ce cas, il est facile de situer la dent du peigne la plus proche de $v_{c w}$. Dans le cas où cette connaissance $a$ priorin'est pas suffisante, il est tout de même relativement aisé de déterminer l'entier $\mathrm{N}_{\mathrm{cw}^{\prime}}$ en effectuant plusieurs mesures successives à plusieurs cadences de répétition du peigne $f_{\text {rep }}$ différentes, et en imposant que les résultats de ces mesures doivent être identiques (i.e. en supposant que la fréquence $v_{\mathrm{cw}}$ n'évolue que de façon négligeable entre les mesures successives). De même, certaines indéterminations de signes pouvant apparaître dans la relation arithmétique liant les paramètres $\mathrm{f}_{\mathrm{b}, \mathrm{Ncw}^{\prime}} \mathrm{f}_{\text {rep, }} \mathrm{f}_{0}$ et $\mathrm{v}_{\mathrm{cw}}\left(\right.$ et $\mathrm{N}_{\mathrm{cw}}$ ) sont aisément levées en étudiant les sens de variation de certains paramètres laissés libres quand on en force d'autres à bouger de manière contrôlée.

Les détails pratiques de l'utilisation du peigne de fréquence et de la relation arithmétique entre ses différents paramètres peuvent différer d'un utilisateur ou d'un laboratoire à un autre en fonction des objectifs, des habitudes ou des détails pratiques d'implémentation. On pourra par exemple asservir en phase certains des

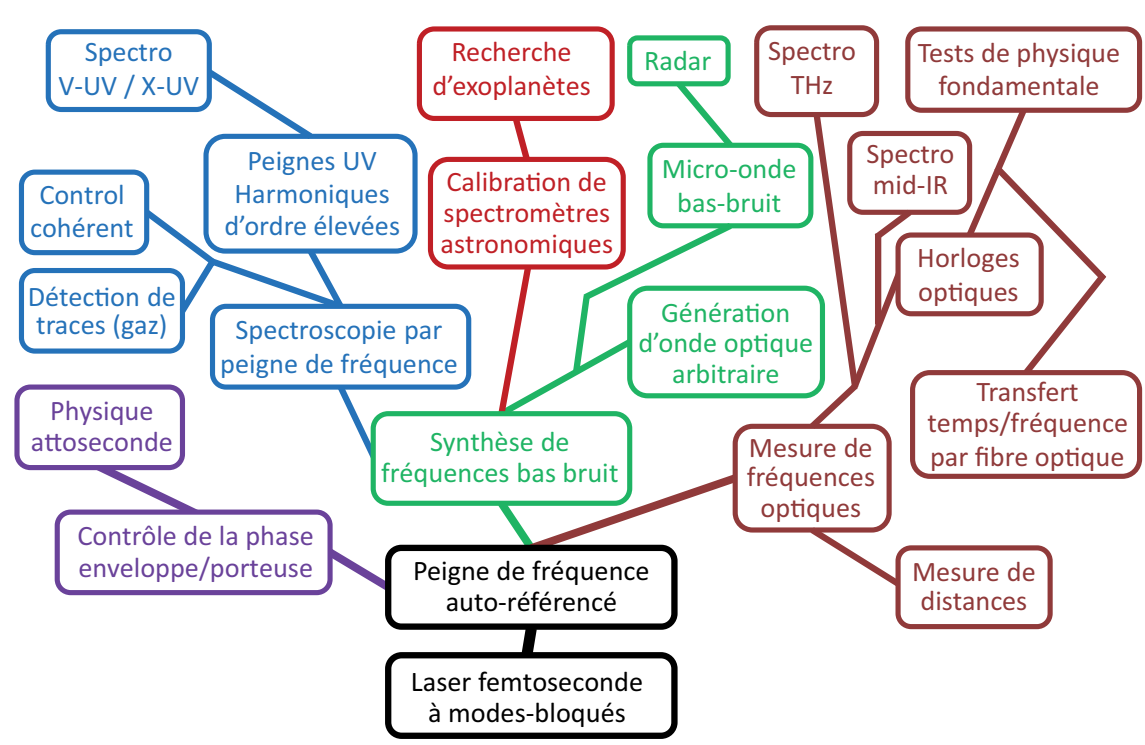

Figure 4. "Arbre évolutif » des peignes de fréquences auto-référencés. En pointillé, on a représenté des domaines d'applications futurs fortement envisagés mais encore tout juste émergents. Figure inspirée de l'article [2], avec l'aimable autorisation de l'auteur.

paramètres $\mathrm{f}_{\mathrm{b}, \mathrm{Ncw}} \mathrm{f}_{\mathrm{rep}}$ et $\mathrm{f}_{0}$ en les comparant à des signaux issus de synthétiseurs RF à fréquence fixe ou variable et mesurer les autres à l'aide de compteur de fréquences, ou encore faire des combinaisons habiles (en hardware ou software) de certains de ces signaux RF pour supprimer tel ou tel paramètre des équations, voire combiner plusieurs de ces approches en même temps. Dans tous les cas, le paramètre le plus fondamental pouvant limiter les performances est le rapport signal à bruit qu'il est possible d'obtenir sur chacun des battements RF considérés, ce pourquoi un montage optique très soigné est le prérequis d'une excellente performance de mesure.

Les performances en termes de précision de mesure (on parle plutôt, en métrologie, de stabilité - liée au bruit, et d'exactitude - liée aux biais résiduels) de ces systèmes sont époustouflantes ! Des bruits de mesure relatifs de $10^{-16}$ à $10^{-18}$ en 1 seule seconde de moyennage sont démontrés régulièrement en laboratoire, avec des erreurs résiduelles associées inférieures à $10^{-19}$ voire $10^{-20}$. De telles performances vont bien au-delà de ce que les horloges atomiques elles-mêmes sont aujourd'hui capables de produire, et il faudra de nombreuses années avant que les peignes de fréquences ne puissent constituer une limitation aux systèmes de métrologie des fréquences optiques considérés dans leur ensemble.

\section{Au-delà de la métrologie pure}

Nés des besoins de mesure de fréquences optiques dans les laboratoires métrologiques, les peignes de fréquences ont vu, depuis, I'apparition de nombreuses recherches pour leur trouver des applications autres. Celles-ci mettent à profit leur précision extrême, leur facilité d'utilisation et leurs coûts d'achat et d'entretien comparativement modiques. Si une liste exhaustive est bien difficile à établir - elle évolue d'année en année au demeurant on pourra citer en particulier la calibration de spectromètres de haute précision pour l'astronomie, la génération de signaux micro-ondes spectralement très purs pour applications radars, la synchronisation de grands instruments (ensemble de radiotélescopes, accélérateurs de particules...) ou l'extension à des gammes spectrales non-visible (TeraHerz, mid-IR, XUV) pour la spectroscopie fine de divers composés (figure 4). Gageons que la popularisation croissante de ces outils continuera, dans I'avenir à leur trouver de nouveaux débouchés prometteurs.

\section{Références}

[1] T. Hänsch, J. Hall, Nobel Lectures (2005)

[2] S. Diddams, "The evolving optical frequency comb", Journal of the Optical Society of America B, Vol. 27, Issue 11, pp. B51-B62 (2010) 